\title{
Dosimetric Impact of Inter-Fraction Variation in Interstitial HDR Brachytherapy
}

\author{
Saravanan Kandasamy ${ }^{1 *}$, K. S. Reddy ${ }^{1}$, Vivekanandan Nagarajan ${ }^{2}$, \\ Parthasarathy Vedasoundaram ${ }^{1}$, Gunaseelan Karunanidhi ${ }^{1}$ \\ ${ }^{1}$ Department of Radiotherapy, Regional Cancer Centre, Jawaharlal Institute of Postgraduate Medical Education \\ and Research (JIPMER), Puducherry, India \\ ${ }^{2}$ Medical Physics Department, Cancer Institute (WIA), Chennai, India \\ Email: "kandasamysaravanan@yahoo.com
}

Received August 9, 2013; revised September 10, 2013; accepted October 1, 2013

Copyright (C) 2013 Saravanan Kandasamy et al. This is an open access article distributed under the Creative Commons Attribution License, which permits unrestricted use, distribution, and reproduction in any medium, provided the original work is properly cited.

\begin{abstract}
Background: Patient setup errors in External Beam Radiotherapy (EBRT) are minimized to a great extent, due to recent technological developments, but in contrary brachytherapy received less attention in inter-fraction catheter movement and its impact in dose delivery. This article deals with inter-fraction interstitial catheter movement and its impact in dose delivery to the target. An attempt is made to study the dosimetric impact of this variation. Objectives: The objective of the study is to evaluate the inter-fraction variation in the position of implanted interstitial applicators and to assess the dosimetric impact in interstitial High Dose Rate (HDR) brachytherapy. Materials and Methods: 55 patients treated for carcinoma tongue, breast, buccal mucosa, cervix, floor of the mouth and soft tissue sarcoma over a period of 2 years (December 2011-May 2013) were considered. All the patients underwent CT scan the next day of the implanting and 3D planning was done either by Eclipse or Oncentra Master Plan Treatment Planning System (TPS). Patients were treated by HDR brachytherapy remote after-loading units, either by Gamma Med iX plus or Microselectron. At the end of the last fraction, CT scan was repeated and re-planning done. The variation in the position of the implanted applicators/catheters and its impact on dosimetric parameters was evaluated and analyzed. Results: The range of positional displacement of the interstitial catheters ranges from $4.5 \mathrm{~mm}$ to $6.8 \mathrm{~mm}$. The maximum variation in prescribed dose to D90 of Clinical Target Volume was 10.88\%. Conclusions: If the total duration of the interstitial implant of HDR brachytherapy extends for more than a week from the day of imaging, it is recommended to do CT imaging and re-plan again. It is mandatory to suture the buttons of the implanting to the skin. Edema and movement of organs (e.g., tongue) are the main cause for the positional variation of the catheters.
\end{abstract}

Keywords: HDR; Brachytherapy; Interstitial Implant; Inter-Fraction Variation; Dosimetric Variation

\section{Introduction}

The worldwide incidence of squamous cell carcinoma of head and neck is more than 500,000 cases per year and the management of patients with head and neck cancer is complex [1]. The choice of treatment modality depends on the stage and site of the disease. Brachytherapy plays an integral role in the management of head and neck cancers and has been described as the first form of conformal radiation [2]. Precise source placement enables very high doses within the tumor and sufficient dose at the margin between the tumor and normal tissue ensuring high tumor control. At the same time, only small vol-

*Corresponding author. umes of normal tissue are irradiated thus decreasing the normal tissue complications.

Brachytherapy involves the implantation of plastic catheters (applicators) into tumors. In 1904, Wickham and Derais used sharpened goose quills to perform intratumoral implantations [3]. Abbe and Morton have reported anecdotal reports of cure for cancers in the head and neck with brachytherapy [4,5]. In 1909, Minet described the first use of a radium tube placed in a catheter to treat prostate cancer [6]. Traditionally treatment planning of brachytherapy was mainly based on radiographs and point dosimetry [6]. The dose distribution was related to the geometry of the catheters. With the newer three dimensional treatment planning together with CT 
imaging, it is possible to get a $3 \mathrm{D}$ based dose distribution with reconstruction of the tumor volume and the catheters [7].

Interstitial implant procedure involves in using a metal needle or trocar. The skin is pierced at the planned entry site and coursed along in the tumor volume, exiting at the marked skin site at the other end of the target volume. Once in place, a nylon after-loading catheter is then adjusted such that the wider portion of the after-loading catheter encompasses the target volume. The trocar is then removed along its original pathway while holding the implanted catheter in place. A metal button along with a half-moon-shaped plastic button is then threaded over the exposed ends of the catheter and crimped in placed over the skin entry sites. Silk ties can then be sutured to the skin through the available holes in the button for further stabilization.

The exposed end of the catheter is then cut off leaving at least several centimeters distal to the metal button. These series of steps would then be repeated, resulting in a parallel distribution of catheters along the tumor volume (Figure 1). Depending upon the site, a patient's comorbidities and extent of implanting, the procedure can be done under local or general anaesthesia. Since brachytherapy treatment is delivered over many days, the implanted catheters can move either due to oedema or movement of the implanted organ.

The positional stability of the catheters and the resultant dosimetric variation over a period of time is studied and presented.

\section{Objectives}

The objective of the study is to quantify the variation in the position of implanted applicators during treatment and assess the dosimetric impact of this variation on Clinical Target Volume (CTV) in interstitial HDR brachytherapy.

\subsection{Materials and Methods}

The remote after-loading HDR Brachytherapy treatment unit GammaMed plus iX plus (Varian Medical Systems, Palo Alto, CA) or Microselectron HDRV3 (Nucletron, BV) using single sealed Iridium 192 radioactive source was used. For treatment planning Eclipse (Varian Medical Systems, Palo Alto, CA) or Oncentra Master plan (Nucletron, BV) was used. Images for planning were acquired by CT Somatom spirit (Siemens). Fifty five patients were included in this study from December 2011 to May 2013 (Table 1). The patients were treated after evaluation according to the stage of the disease.

\subsection{Treatment Protocol}

Patients were treated according to the institutional pro-

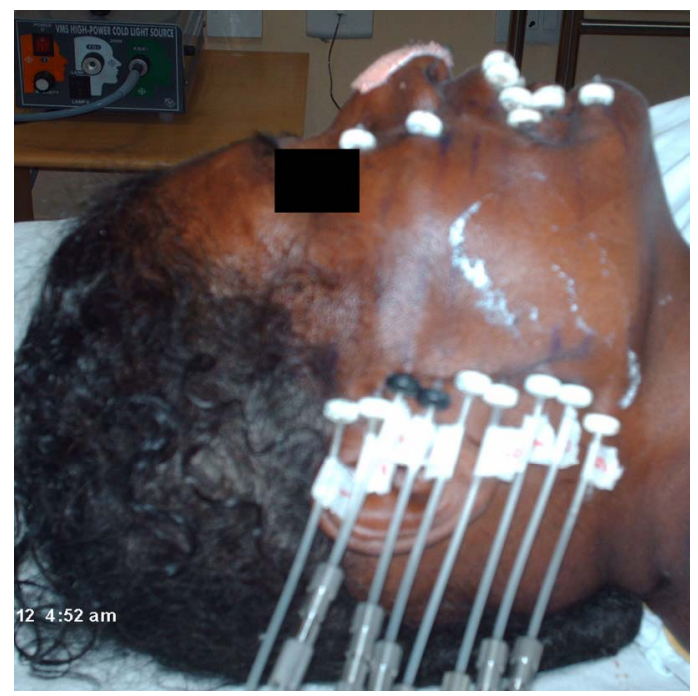

Figure 1. Patient with flexible interstitial implant.

Table 1. Patient characteristics.

\begin{tabular}{|c|c|}
\hline Characteristics & No of Patients (\%) \\
\hline \multicolumn{2}{|l|}{ Age } \\
\hline Mean & 50.1 \\
\hline Standard Deviation & 10.3 \\
\hline Median & 48 \\
\hline Range & $32-73$ \\
\hline \multicolumn{2}{|l|}{ Gender } \\
\hline Male & $26(47.27 \%)$ \\
\hline Female & $29(52.72 \%)$ \\
\hline \multicolumn{2}{|l|}{ Diagnosis } \\
\hline Carcinoma Breast & $13(23.63 \%)$ \\
\hline Carcinoma Buccal Mucosa & $17(30.91 \%)$ \\
\hline Carcinoma Cervix & $1(1.82 \%)$ \\
\hline Carcinoma Floor of Mouth & $2(3.64 \%)$ \\
\hline Carcinoma Tongue & $19(34.55 \%)$ \\
\hline Soft Tissue Sarcoma (Multiple Site) & $3(5.45 \%)$ \\
\hline \multicolumn{2}{|l|}{ T Stage* } \\
\hline $\mathrm{T} 1$ & $6(10.91 \%)$ \\
\hline $\mathrm{T} 2$ & $20(36.36 \%)$ \\
\hline $\mathrm{T} 3$ & $29(52.73 \%)$ \\
\hline $\mathrm{T} 4$ & $0(0 \%)$ \\
\hline \multicolumn{2}{|l|}{ N Stage ${ }^{*}$} \\
\hline N0 & $42(76.36 \%)$ \\
\hline N1 & $10(18.18 \%)$ \\
\hline $\mathrm{N} 2$ & $3(5.45 \%)$ \\
\hline N3 & 0 \\
\hline
\end{tabular}

*According to the 7th American Joint Commission on Cancer/Union for International Cancer Control Staging system. 
Table 2. Institutional treatment protocol.

\begin{tabular}{|c|c|c|}
\hline Diagnosis & EBRT $^{*}$ & HDR $^{* *}$ \\
\hline Carcinoma & 200 cGy x & 250 cGy x \\
\hline Breast & 20 fractions & 6 fractions \\
\hline Carcinoma & 200 cGy x & 350 cGy x \\
\hline Buccal Mucosa & 25 fractions $^{* * *}$ & 6 fractions \\
\hline Carcinoma & 200 cGy x & 400 cGy x \\
\hline Cervix & 25 fractions & 5 fractions \\
\hline Carcinoma & 200 cGy x & 350 cGy x \\
\hline Floor of Mouth & 25 fractions $^{* * *}$ & 6 fractions \\
\hline Soft Tissue & 200 cGy x & 250 cGy x \\
\hline sarcoma & 20 fractions & 6 fractions \\
\hline Carcinoma & 200 cGy x & 350 cGy x \\
\hline Tongue & 25 fractions $^{* * *}$ & 6 fractions \\
\hline
\end{tabular}

${ }^{*}$ Five fractions per week with one fraction per day. ${ }^{* *}$ Two fractions per day with 6 hours gap between the two fractions. ${ }^{* * *}$ With Spinal shield after 44 Gy.

tocol (Table 2).

\subsection{Interstitial Implant Application}

Under general anaesthesia, trocars and hollow needles were inserted as guide tubes in and around the tumor 1 $\mathrm{cm}$ apart in single or multiple planes through which plastic tubes were threaded. Theses tubes were then secured by buttons. Similarly for rigid needle implant, the sterilized needles with the appropriate length were selected. With the guidance of templates the needles were inserted into the tissue. The template helped to maintain a proper geometry of the needle placement. The needles were secured by stainless steel buttons. Table 3 gives the details of the interstitial implants.

\subsection{Imaging and Planning}

On the second day of implantation, the patients underwent CT scan of the involved region with a slice thickness of $1 \mathrm{~mm}$. The applicator reconstruction was done and at the tip of all the applicators a reference point was inserted (Figure 2). The source dwell positions and step size were identified and accordingly the fine tuning of dose optimization was performed by changing the dwell time and dwell weight age for individual dwell positions. In most cases, dwell time was changed to reduce the hot spot or to remove the cold spot. Graphical optimization was never used. It was ensured that at least $90 \%$ of the CTV receives the prescribed dose. The dose distribution was generated by TPS using the AAPM TG-43 dose formalism [5]. Treatment was delivered using the HDR remote after-loading system. On the last fraction a repeat $\mathrm{CT}$ and re-planning was done and the catheters were removed.

For each patient planning was done on both Pre HDR and Post HDR images. Pre HDR and Post HDR images were fused and matched using a prominent anatomical land mark. The step size, dwell position and dwell time was maintained the same in both the plans and only the
Table 3. Institutional treatment protocol.

\begin{tabular}{cc}
\hline Type & No of Patients (\%) \\
\hline Rigid Needle Implants & $13(23.64 \%)$ \\
Flexible Catheter Implants & $42(76.36 \%)$ \\
Number of Planes & \\
Single Plane & $17(30.91 \%)$ \\
Double Plane & $34(61.82 \%)$ \\
Triple Plane & $4(7.27 \%)$ \\
\hline
\end{tabular}

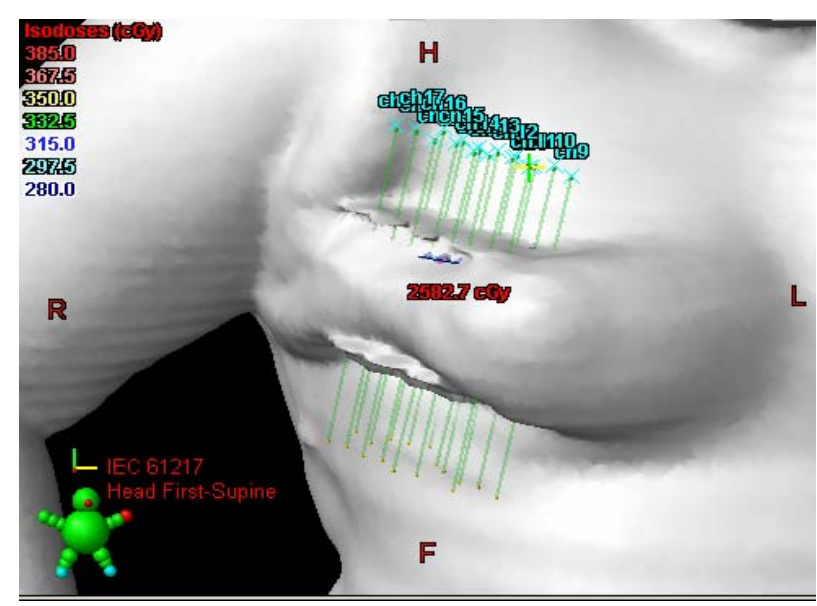

(a)

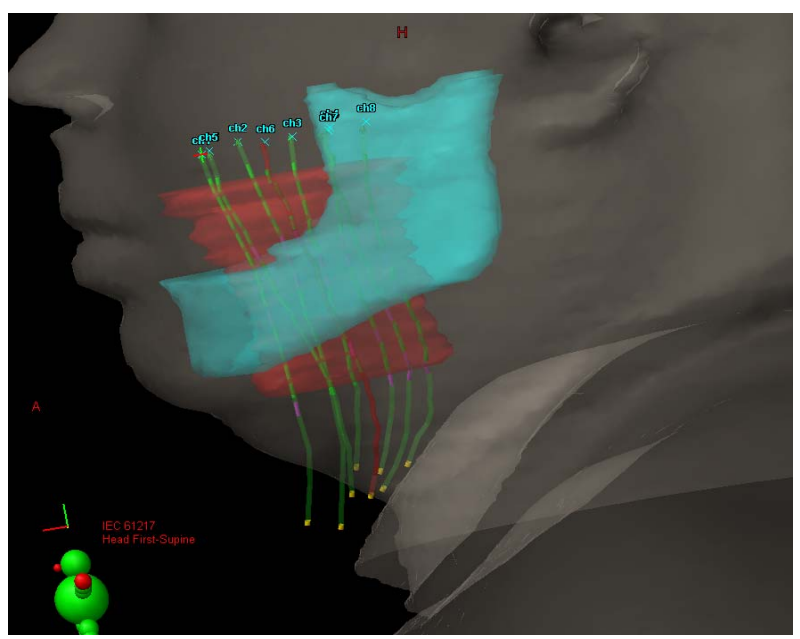

(b)

Figure 2. (a) Reconstructed rigid needle implant with reference points-Cancer Right Breast; (b) Reconstructed flexible implant with reference points-Cancer Left Tongue.

catheter position was updated in the post HDR brachytherapy plan. The tip of the catheters where the referencepoints were inserted gave the co-ordinates in $\mathrm{x}, \mathrm{y}$ and $\mathrm{z}$ axis. The variation in the reference points between the two plans were estimated which gave the actual displacement in the catheter position in 3D axis. Using the Dose Volume Histogram (DVH) the dosimetric parameters were studied. 


\section{Result}

A total of 55 patients were treated between December 2011 to May 2013. 110 brachytherapy plans were generated. The demographic details of the patients included in the study are listed in Table 1. In three patients, brachytherapy was the sole modality of treatment. All other patients were treated with both external beam radiation and brachytherapy.

Table 4 gives the details of the mean dose variation (\%) to D90 of CTV. Rigid implant was used for patients with carcinoma breast and cervix $(25.45 \%)$. For all the other patients flexible catheter implants were used $(74.55 \%)$. Table 5 gives the variation in dose to the volume receiving $150 \%$ of the prescribed dose.

For patients with carcinoma breast the displacement in catheter position is shown in Figure 3. For all the patients the catheter displacement and D90 dose to CTV is less than $3 \mathrm{~mm}$ and $3 \%$ respectively.

For patients with carcinoma buccal mucosa the catheter displacement for $35.29 \%$ of the patients was more than $5 \mathrm{~mm}$ (Figure 4). In $29.41 \%$ of the patients D90 dose to CTV was more than $3 \%$.

For patients with carcinoma tongue the displacement in catheter position is shown in Figure 5. The catheter displacement for $42.11 \%$ of patients was more than $5 \mathrm{~mm}$. As per DVH in $26.32 \%$ of the patients D90 dose to CTV was more than $3 \%$.

Figure 6 shows the catheter displacement in patients with soft tissue sarcoma ( 3 patients), carcinoma floor of mouth ( 2 patients) and carcinoma cervix (1 patient).

In Figure 7 the maximum dosimetric variation (D90) to CTV is shown with respect to the time interval in days between the Pre HDR and Post HDR plans. In 15 patients the duration between the two plans was 5 days, in 10 patients it was 6 days. In 5 patients the time interval was 7 days and in 8 patients it was 8 days. 5 patients $(9.09 \%)$ had 9 days and 2 patients $(3.67 \%)$ had 10 days interval between the plans. The variation in intervals between plans was because of treatment protocol, holidays and machine down time.

\section{Discussion}

Feng $\mathrm{Xu}$ et al. evaluated interfractional and intrafrac

Table 4. Dosimetric variation in percentage to D90 of CTV.

\begin{tabular}{cc}
\hline Type of Tumor & Mean Value + 1 Standard Deviation \\
\hline Carcinoma Breast & $0.423 \pm 1.72$ (13 Patients) \\
Carcinoma Buccal Mucosa & $1.16 \pm 3.88$ (17 Patients) \\
Carcinoma Tongue & $1.22 \pm 2.71$ (19 Patients) \\
Soft Tissue Sarcoma & $1.53 \pm 4.66$ (3 Patients) \\
Carcinoma Floor of Mouth & $4.6 \pm 5.37$ (2 Patients) \\
Carcinoma Cervix (MUPIT) & 5.72 (1 Patient) \\
D90-Dose Received by at Least $90 \%$ of the Volume.
\end{tabular}

Table 5. Dosimetric variation to volume receiving $150 \%$ of dose.

\begin{tabular}{ccc}
\hline Type of Tumor & \multicolumn{2}{c}{$\begin{array}{c}\text { Variation in Volume Receiving } \\
\text { More than 150\% of Dose }\end{array}$} \\
\hline Carcinoma Breast & $1.8 \mathrm{~cm}^{3}$ & $(1.76 \%)$ \\
Carcinoma Buccal Mucosa & $-6.42 \mathrm{~cm}^{3}$ & $(-2.14 \%)$ \\
Carcinoma Tongue & $1.04 \mathrm{~cm}^{3}$ & $(7.91 \%)$ \\
Soft Tissue Sarcoma & $1.3 \mathrm{~cm}^{3}$ & $(2.31 \%)$ \\
Carcinoma Floor of Mouth & $2.8 \mathrm{~cm}^{3}$ & $(2.85 \%)$ \\
Carcinoma Cervix (MUPIT) & $1.2 \mathrm{~cm}^{3}$ & $(1.35 \%)$ \\
\hline
\end{tabular}

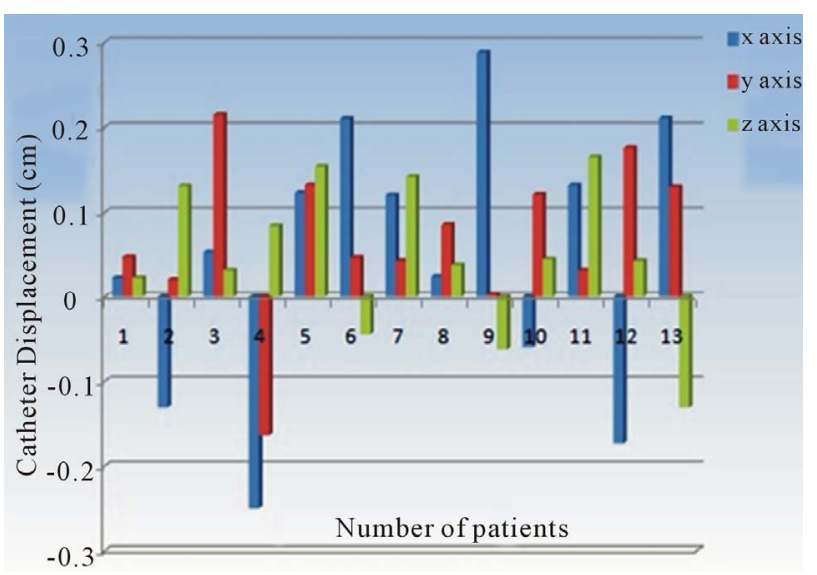

Figure 3. Catheter displacement for carcinoma breast.

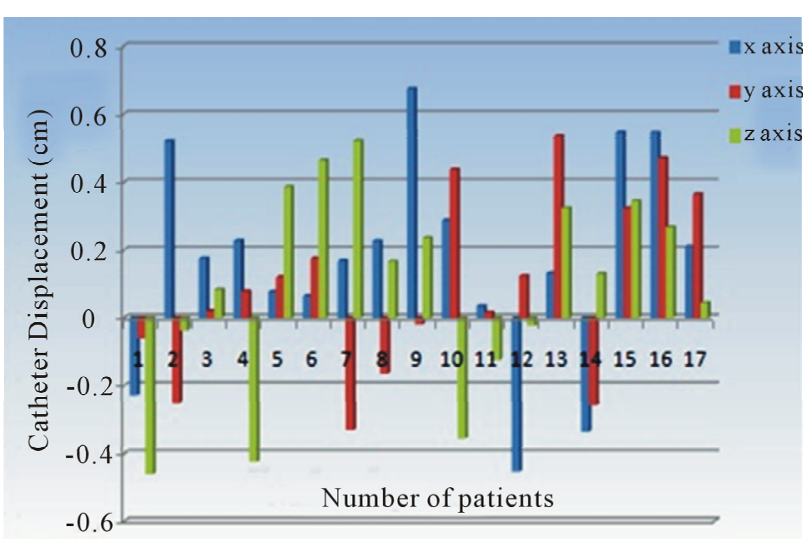

Figure 4. Catheter displacement for carcinoma buccal mucosa.

tional setup error in external beam radiotherapy using cone beam computed tomography as per their result a total of 1934 CBCT scans were performed on 51 patients, the setup errors were $\leq 2 \mathrm{~mm}$ on all axes [8] similarly Slow T. R. et al. has estimated interfraction prostate motion during intensity modulated radiotherapy for prostate cancer of 157 patients with prostate cancer the mean setup errors $21 \mathrm{~mm}$ [9], whereas in interstitial HDR Brachytherapy we have estimated that the catheter positional variation is $>6 \mathrm{~mm}$ for few cases which was not 


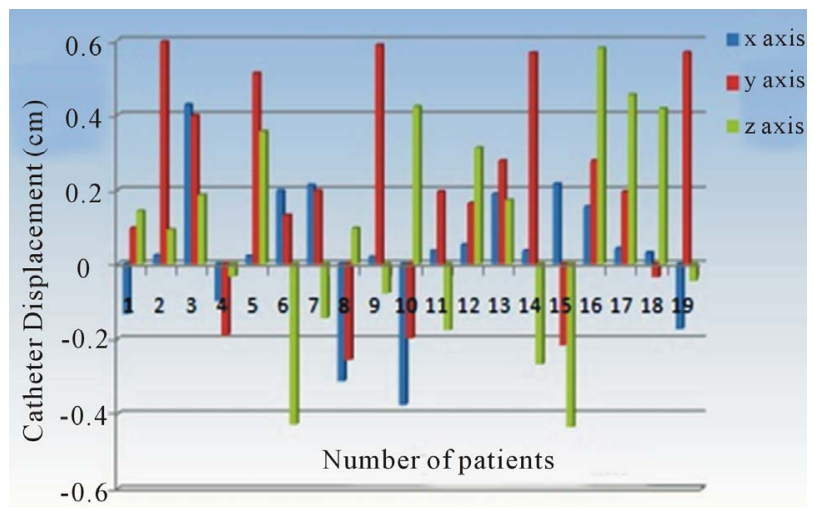

Figure 5. Catheter displacement for Carcinoma Tongue.

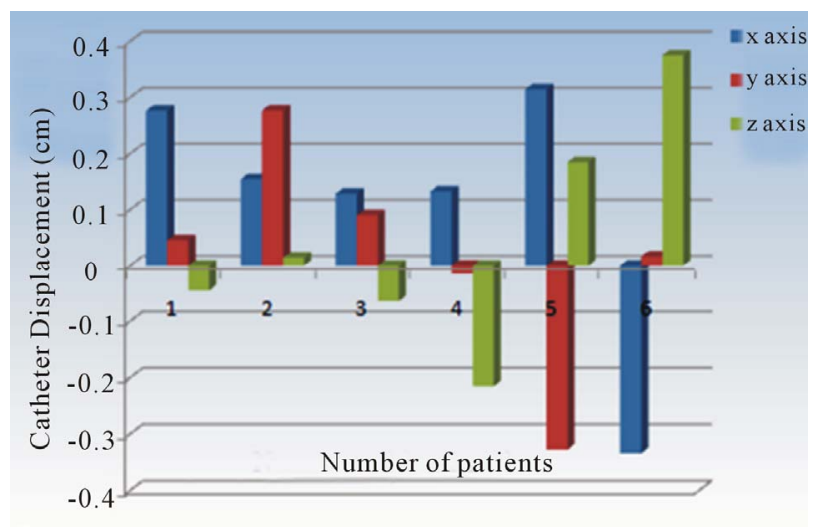

Figure 6. Catheter displacement for soft tissue sarcoma (1 3 ), carcinoma floor of mouth $(4,5)$ and carcinoma cervix $(6)$.

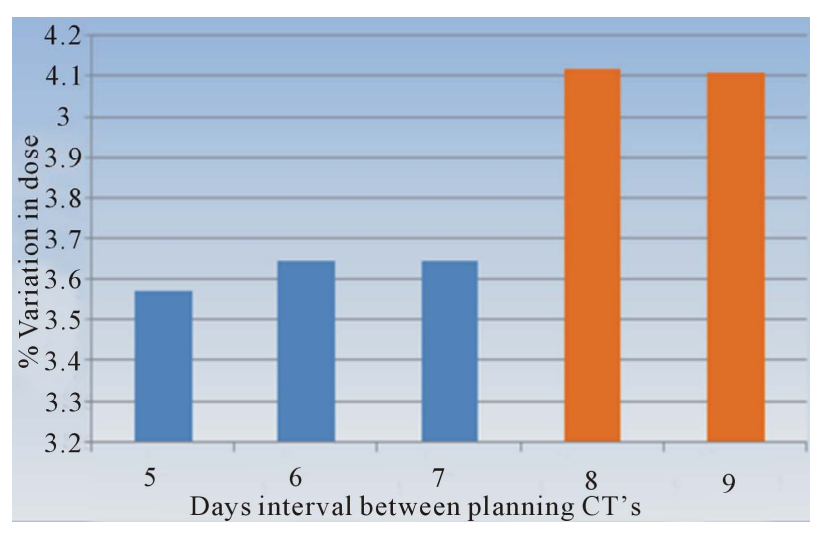

Figure 7. Dosimetric variation with time.

taken into account seriously. Velumurugan et al. evaluated MUPIT implantation in patients with carcinoma cervix. In their study they recommended to repeat CT and re-plan before each fraction [10]. In our study imaging and planning was done before the first fraction. This plan was used for treatment delivery. At the end of the last fraction imaging and re-planning was done again and this plan was used only for analysis. Velumurugan et al. observed that the CTV mean dose varies from $+9.8 \%$ to $-13.3 \%$. Out of 10 patients the mean dose was more than the prescribed dose in seven patients and less in three patients [10]. The maximum variation to CTV dose was estimated to be around $13 \%$. In majority of the cases the variation was around $1 \%$. However in their study the physical movement of the needles was not estimated. Similarly Rey F., et al. in their study estimated the day 1 plan on the day 2 and day 3. CT and re-plan was done before each treatment, with updated catheter positions the treatment was delivered [8]. The mean CTV D90 was reduced from $93.4 \%$ on day 1 to $89.3 \%$ on day 2 and to $87.7 \%$ on day 3 . Re-planning on day 2 and day 3 compensated for catheter movement. They have also not estimated the positional variation of catheters. In our study we have estimated the positional variation of catheters and its dosimetric implant and we have also extended the study to different sites of implants and given the comparison. Similarly we have also given the comparison between rigid and flexible implants. As per International Commission on Radiation Units and Measurements (ICRU 58) the dosimetric variation should be limited to $3 \%$ of D90 to CTV [11]. As per our results it has been identified that for carcinoma tongue the variation in catheter dislocation is relatively more when compared to other sites because of the movement of the organ. Hence, dosimetric variation is more for implants of carcinoma tongue. Our data also shows those rigid needle implants are more stable compared to flexible implant with less dosimetric variation for the former.

\section{Conclusion}

An inter-fraction error is reported frequently in HDRbrachytherapy. Rigid needle implants are dosimetrically superior to flexible implants. Suturing of the buttons in flexible implanting is not a solution for arresting the catheter movement, since it immobilizes the button alone and the catheter movement is still possible. Adequate care should be taken while connecting the catheters to the HDR brachytherapy unit with transfer tubes to prevent physical displacement of the catheters. With an increase in duration of treatment, the inter-fraction error increases and it is recommended to repeat CT and re-plan on the fifth day from the day of implanting.

\section{REFERENCES}

[1] R. Ortega, A. Loria and R. Kelly, "A Semiglobally Stable Output Feedback PI2D Regulator for Robot Manipulators," IEEE Transactions on Automatic Control, Vol. 40, No. 8, 1995, pp. 1432-1436. http://dx.doi.org/10.1109/9.402235

[2] A. Jemal, T. Murray, A. Samuels, A. Ghafoor, E. Ward and M. J. Thun, "Cancer Statistics 2003," A Cancer Journal for Clinicians, Vol. 53, No. 1, 2003, pp. 5-26. http://dx.doi.org/10.3322/canjclin.53.1.5 
[3] R. G. Dale and B. Jones, "The Clinical Radiobiology of Brachytherapy," British Journal of Radiology, Vol. 71, No. 845, 1998, pp. 465-483.

[4] C. A. Joslin, A. Flynn and E. J. Hall, "Principles and Practice of Brachytherapy Using Afterloading Systems," Arnold, London, 2001.

[5] E. K Salminen, K. Kiel, G. S. Ibbott, M. C. Joiner, E. Rosenblatt, E. Zubizarreta, et al., International Conference on Advances in Radiation Oncology (ICARO): Outcomes of an IAEA Meeting, Vienna, 27-29 April 2009. http://dx.doi.org/10.1186/1748-717X-6-11

[6] R. Nath, L. L. Anderson, G. Luxton, K. A. Weaver, J. F. Williamson and A. S. Meigooni, "Dosimetry of Interstitial Brachytherapy Sources: Recommendations of the AAPM Radiation Therapy Committee Task Group No. 43. American Association of Physicists in Medicine," Medical Physics, Vol. 22, No. 2, 1995, pp. 209-234. http://dx.doi.org/10.1118/1.597458

[7] F. Rey, C. Chang, C. Mesina, N. Dixit, B. K. Kevin Teo and L. L. Lin, "Dosimetric Impact of Interfraction Catheter Movement and Organ Motion on MRI/CT Guided HDR Interstitial Brachytheratpy for Gynecologic Can- cer," Radiotherapy \& Oncology, Vol. 107, No. 1, 2013, pp. 112-116.

http://dx.doi.org/10.1016/j.radonc.2012.12.013

[8] F. Xu, J. Wang, S. Bai, Q.-F. Xu, Y.-L. Shen and R.-M. Zhong, "Interfractional and Intrafractional Setup Errors in Radiotherapy for Tumors Analyzed by Cone-Beam Computed Tomography," Chinese Journal of Cancer, Vol. 27, No. 10, 2008, pp. 373-376.

[9] T. R. Slow, C. L. F. Ngol and W. K. T. Tan, "Inter-Fraction Prostate Motion during Internsity-Modulcated Radiotherapy for Prostate Cancer," Singapore Medical Journal, Vol. 52, No. 6, 2011, p. 405.

[10] T. Velmurugan, P. Sukumar, C. Krishnappan and R. Boopathy, "Study of Dosimetric Variation Due to Interfraction Organ Movement in High Dose Rate Interstitial (MUPIT) Brachytherapy for Gynecologic Malignancies," Polish Journal of Medical Physics and Engineering, Vol. 16, No. 2, 2010, pp. 85-95.

[11] International Commission on Radiation Units and Measurements (ICRU), "Report 58 on Dose Volume Specification for Reporting Interstitial Therapy," ICRU, Bethesda, 1997, pp. 1-45. 